\title{
Impacto de variables socioeconómicas en el desempeño estudiantil
}

\section{Impact of socioeconomic variables on student performance}

Manuel Arturo Jiménez Ramirez
manuel.jimenezr@upb.edu.co

Marianela Luzardo Briceño ${ }^{c}$

marianela.luzardo@upb.edu.co

\author{
Edward Parra ${ }^{\mathrm{b}}$ \\ edward.parra@javeriana.edu.co
}

\section{Resumen}

Se pretende identificar elementos explicativos a la variabilidad observada en los resultados obtenidos de las pruebas Saber Pro en los módulos de Comunicación Escrita, Inglés, Lectura Crítica, Razonamiento Cuantitativo y Competencias Ciudadanas a partir de las bases de datos suministradas por el Instituto Colombiano para el Fomento de la Educación Superior en el periodo comprendido entre 2012-10 y 2015-30 de los estudiantes de la Universidad Pontificia Bolivariana de Colombia. Se analizaron variables de tipo binario correspondientes a la existencia o no de recursos como internet, televisión, telefonía, lavadora, horno, microondas, nevera, automóvil y reproductor DVD en los hogares en los que reside el estudiante. En virtud de la gran cantidad de datos con la que se cuenta se seleccionó la técnica de Clúster Bietápico para conocer la agrupación de la información. Con éstos se realizó un paralelo con los puntajes obtenidos en la prueba Saber Pro y se encontró que, en su mayoría, los estudiantes con mayores recursos económicos obtienen los mejores puntajes en las Pruebas en cuestión.

\begin{abstract}
This document aims to identify explanatory elements to the variability observed in the results of the Saber Pro tests in the modules of Written Communication, English, Critical Reading, Quantitative Reasoning and Citizen Competences from databases supplied by the Colombian Institute for the Promotion of Higher Education Institute in the period between 2012-10 y 2015-30 of students of Pontifical Bolivarian University. It was analyzed variables of binary type corresponding to
\end{abstract}

\footnotetext{
aniversidad Pontificia Bolivariana, Bucaramanga

b Pontificia Universidad Javeriana

${ }^{\mathrm{c}}$ Universidad Pontificia Bolivariana Bucaramanga
} 
the existence or not of resources such as Internet, Television, Telephony, Washing Machine, Oven, Microwave, Fridge, Car, and DVD Player in the homes in which the student resides. In virtue of the large amounts of data the two-step-cluster analysis technique was selected to know the grouping of the information. Whith these results a parallel was made with the scores obtained in the Saber Pro test and it was found that, mostly, the students with more economic resources obtain better scores in the test.

Keywords: Cluster analysis, Classification methods, Analysis of Education, Social and Economic Stratification..

\section{Introducción}

La autoevaluación institucional con fines de acreditación está adaptando nuevas formas de evaluación, obligando a las instituciones a redefinir sus procesos académicos y curriculares con el fin de favorecer la equidad en la enseñanza de las diferentes disciplinas. Según Field et al. (2007) la equidad hace referencia a hacer posible que la mayoría de estudiantes desarrollen competencias y rindan bien en las instituciones, así como adquiriendo habilidades básicas y a realizarse como seres humanos, ya que dentro de los pilares se encuentra en impacto social que generan las instituciones. Field et al. (2007) arguye que equidad en educación incluye dos dimensiones, justicia e inclusión: Justicia, implica que las circunstancias personales y sociales como el género, estatus socioeconómico u origen étnico no deberían ser un obstáculo para el éxito educativo; inclusión, implica un estándar mínimo de educación para todos.

Una de las últimas metas de los diseñadores de políticas públicas es la de permitir a los ciudadanos tomar ventaja de una economía mundial globalizada; esto los está guiando a enfocarse en la mejora de las políticas educativas, asegurando la calidad en la prestación del servicio, una mayor distribución de igualdad de oportunidades de aprendizaje e iniciativas más fuertes para mayor eficiencia en las escuelas (for Economic Co-operation \& (OECD) 2010).

Como lo mencionan Field et al. (2007), equidad es diferente de igualdad. Esta está asociada con ideas más amplias sobre justicia, algunas veces con "igualdad de oportunidad" y otras con "trato equivalente". Para ellos equidad en educación incluye dos dimensiones: justicia e inclusión. Justicia, implica que las circunstancias personales y sociales como el género, estatus socioeconómico u origen étnico no deberían ser un obstáculo para el éxito educativo; inclusión, implica un estándar mínimo de educación para todos.

En este orden de ideas, el estatus socioeconómico es una medida de ventaja relativa, que explica las múltiples características de los antecedentes, incluyendo ingreso familiar, educación y ocupación de los padres.

Brown et al. (2017) en su investigación sobre el estatus socioeconómico de los estudiantes, encontró que solo 1 de cada 10 ellos en condición económica no favorecida 


\section{Manuel Arturo Jiménez Ramirez, Edward Parra \& Marianela Luzardo Briceño}

se ubica en el cuartil más alto de desempeño, comparado con cerca de la mitad de los estudiantes de hogares más privilegiados. Dentro del cuartil más alto de logro, hubo pequeñas diferencias significativas de desempeño (resultado promedio) entre los grupos de estudiantes de estatus socioeconómico alto y bajo. Específicamente, en pruebas de lectura y matemáticas, los estudiantes con alto desempeño y de estatus socioeconómico bajo respondieron cerca de 1.5 de preguntas menos correctamente que los estudiantes de alto desempeño con estatus socioeconómico alto, en promedio.

Malas habilidades básicas significan menos oportunidad de un trabajo, mala salud, más criminalidad y una vida más corta. La evidencia muestra que el riesgo es más agravado para aquellos menos favorecidos y que reciben una educación débil (OECD 2012).

Por supuesto, no todos los estudiantes pueden tener que rendir bien en la escuela, pero la meta de equidad en educación es la de asegurar que tantos, como sea posible, lo hagan - adquirir habilidades básicas y adicionales, realizarse como seres humanos superando accidentes de circunstancias personales y contexto familiarno hay inevitabilidad para el fracaso en la educación. En Finlandia, y a través de otras iniciativas en muchos países diferentes, el fracaso y el abandono escolar puede ser abordado exitosamente (Field et al. 2007).

Reardon (2011) analiza la brecha entre el ingreso familiar y logro académico de los estudiantes, a partir de la consecución de los estudios educativos de los padres, concluyendo que, aunque un aumento en la desigualdad de ingresos puede jugar un rol en el crecimiento de la brecha de desempeño, éste no parece ser un factor dominante. La brecha parece haber crecido, al menos en parte, por un incremento en la asociación ingreso familiar y desempeño académico del estudiante para familias por encima de la media del nivel de ingresos.

En este sentido, las instituciones de educación superior están reconociendo cada vez más que son parte de las industrias de servicios con los estudiantes como el consumidor principal (Alfiani et al. 2015). Uno de los indicadores de desempeño académico estudiantil es el promedio acumulado de los estudiando que dinámicamente va cambiando semestre a semestre (Alfiani et al. 2015).

Mejorar el desempeño académico requiere información relacionada con las condiciones socio-demográficas del estudiante, en virtud que los estudiantes tienen diferentes niveles de motivación, entendimientos de la enseñanza y aprendizaje y, las condiciones de su entorno son diferentes entre unos y otros. Por consiguiente, las técnicas de minería de datos no son solo útiles para mapear a los estudiantes basado en variables demográficas, el entendimiento de proceso de aprendizaje, el nivel de actividad, sino también puede ser utilizada para conocer las variables que influyen en el nivel de desempeño de logro de los estudiantes en las pruebas Saber Pro como el promedio acumulado, puntajes de pruebas, duración del estudio, entre otros. El Instituto Colombiano para el Fomento de la Educación Superior (ICFES), es una entidad pública adscrito al Ministerio de Educación Nacional, que evalúa el sistema educativo colombiano en todos sus niveles y modalidades. Dentro de las pruebas que realiza se encuentran las Saber Pro, a partir de la cual 
se conocen los niveles de competencias específicas y genéricas de los estudiantes universitarios a nivel nacional.

Las pruebas Saber Pro son evaluaciones censales que realiza el Ministerio de Educación para conocer los niveles de competencias de los estudiantes a nivel nacional. Se evalúan tanto competencias específicas como genéricas para los programas académicos que se ofrecen en las instituciones. Para esta investigación, y para poder establecer un comparativo entre programas se utilizaron los puntajes de los módulos genéricos que son: Comunicación Escrita, Inglés, Lectura Crítica, Razonamiento Cuantitativo y competencias ciudadanas.

En este trabajo se analiza si las diferentes variables socioeconómicas, departamento de origen, nivel educativo de los padres y el programa académico según grupo de referencia afectan el puntaje obtenido en las pruebas Saber Pro de los estudiantes de la Universidad Pontificia Bolivariana en el periodo comprendido entre 2012-10 y 2015-30, con la finalidad de tener un amplio panorama de las políticas internas que se deban formular para mejorar la calidad de los egresados.

\section{Materiales y métodos}

El presente es un estudio con enfoque cuantitativo, de tipo descriptivo, correlacional y diseño no experimental. Para esta investigación, y para poder establecer un comparativo entre grupos de referencia, se utilizaron los puntajes de los módulos genéricos de la prueba en cuestión, a saber, Comunicación Escrita, Inglés, Lectura Crítica, Razonamiento Cuantitativo y Competencias Ciudadanas. La base de datos utilizada fue suministrada por el ICFES, de la cual se tomaron 11.554 registros de siete semestres, entre 2012-10 y 2015-30.

Luego de analizar la base de datos se procedió a realizar un análisis clúster para conocer las características particulares que tenían los estudiantes en relación a los resultados en la prueba. En virtud que la base de datos tiene en su mayoría variables de tipo categórico, se utilizaron las variables que contenían información binaria con valores 1 (presencia) y 0 (ausencia) para realizar el análisis clúster de una manera más precisa. Las variables son las siguientes:

A partir de estas variables se procedió a utilizar el análisis de clúster bietápico debido a que es exploratorio y no requiere especificaciones a priori del número de clústeres (Sideridis et al. 2006), reduce el sesgo potencial si se utilizara un solo método (Ferguson et al. 2000), incrementa la validez de la solución (Punj \& Stewart 1983); y asume independencia de las variables de tal forma que, la distribución conjunta multinomial-normal puede ser ubicada tanto en variables categóricas como continuas (Brown et al. 2017).

El algoritmo de clúster bietápico utiliza el logaritmo de máxima verosimilitud, el cual es una medida de distancia basada en la probabilidad (IBM Knowledge Center 2012). Cuando se calcula el logaritmo de la verosimilitud se asumen distribuciones multinomiales para variables categóricas, y también que las variables 
114Manuel Arturo Jiménez Ramirez, Edward Parra \& Marianela Luzardo Briceño

Tabla 1: Variables socieconómicas

\begin{tabular}{ll}
\hline \multicolumn{1}{c}{ Variable } & \multicolumn{1}{c}{ Definición } \\
\hline Econ_sn_celular & El hogar cuenta con celular \\
Econ_sn_internet & El hogar cuenta con conexión a internet \\
Econ_sn_servicio_tv & El hogar cuenta con servicio cerrado de televisión \\
Econ_sn_telefonia & El hogar cuenta con servicio de teléfono fijo \\
Econ_sn_lavadora & El hogar cuenta con lavadora \\
Econ_sn_horno & El hogar cuenta con horno eléctrico o gas \\
Econ_sn_microondas & El hogar cuenta con horno microondas \\
Econ_sn_nevera & El hogar cuenta con nevera o enfriador \\
Econ_sn_automovil & El hogar cuenta con automóvil particular \\
Econ_sn_reproductordvd & El hogar cuenta con reproductor DVD \\
\hline
\end{tabular}

Fuente: Diccionario de variables, ICFES, pruebas Saber Pro.

son independientes entre sí (IBM Knowledge Center 2012). La distancia entre un clúster $j$ y un clúster $i$ está relacionada con la disminución en el logaritmo de verosimilitud a medida que se combinan en un solo clúster, su fórmula está definida por:

$$
d(i, j)=\xi_{i}+\xi_{j}-\xi_{<i \cdot j>}
$$

donde

$$
\xi_{v}=-N_{v}\left(\sum_{k=1}^{K^{A}} \frac{1}{2} \log \left(\hat{\sigma}_{k}^{2}+\hat{\sigma}_{v k}^{2}\right)+\sum_{k=1}^{K^{B}} \hat{E}_{v k}\right)
$$

y

$$
\hat{E}_{v k}=-\sum_{l=1}^{L_{k}} \frac{N_{v k l}}{N_{v}} \log \frac{N_{v k l}}{N_{v}}
$$

Si $\hat{\sigma}_{k}^{2}$ es ignorado en la expresión $\xi_{v}$, la distancia entre los clústeres $i$ y $j$ va a ser exactamente la disminución en el logaritmo de la verosimilitud cuando dos clústeres son combinados; el termino $\hat{\sigma}_{k}^{2}$ es añadido para solucionar el problema causado por $\hat{\sigma}_{v k}^{2}=0$, lo que resultaría en que el logaritmo natural fuera indefinido (IBM Knowledge Center 2012).

La selección del modelo se realizó utilizando el criterio de información bayesiana que se computa de la siguiente forma:

$$
B I C(J)=-2 \sum_{j=!}^{J} \xi_{j}+m_{J} \log (N)
$$

donde

Comunicaciones en Estadística, diciembre 2019, Vol. 12, No. 2 


$$
m_{J}=J\left\{2 K^{A}+\sum_{k=1}^{K^{B}}\left(L_{K}-1\right)\right\} .
$$

El algoritmo clúster utiliza un procedimiento en dos etapas que funciona satisfactoriamente con el método jerárquico. En este sentido, en la primera etapa se calcula el Criterio de Información Bayesiano BIC para cada número de clústerses dentro de un rango específico, y utilizado para encontrar el estimador inicial para cada número de clústeres (IBM Knowledge Center 2012).

En el segundo paso, el estimador inicial es redefinido encontrando el incremento relativo más grande en distancia entre en los dos clústeres más cercanos en cada etapa del análisis de clúster jerárquico (IBM Knowledge Center 2012).

El $B I C$ es uno de los criterios más populares para la selección de modelos, tal como lo menciona (Zhao et al. 2015), pero suele penalizar la complejidad de cada componente utilizando todo el tamaño de muestra; la solución a este problema se encuentra en el uso del análisis de clúster jerárquico en la segunda etapa, la cual tiene como clave que los parámetros son penalizados únicamente en base a sus tamaños de muestra efectivos relevantes.

Para realizar el análisis clúster se utilizó el software SPSS Modeler. Las variables seleccionadas (tabla 1 ) se procesaron siguiendo el algoritmo bietápico, con el $B I C$ como criterio de selección del número de clústeres.

Una vez obtenidos los clústeres se realizó un análisis descriptivo de la información para cada uno de ellos en relación con: módulo de referencia, semestre en que presentó la prueba, estrato socioeconómico, departamento de origen, nivel de educación del padre, nivel de educación de la madre, género e institución para conocer el desempeño entre y al interior de cada clúster. La comparación se realizó utilizando como categoría de referencia el clúster 1.

En una segunda fase se utilizó la regresión con variables categóricas para analizar la existencia de diferencias significativas del puntaje por los módulos genéricos que evalúa la prueba. Los modelos de regresión están realizando una prueba de comparación de medias a través de la utilización de variables dummy para cada una de las categorías en cada variable. Para conocer si la diferencia en las medias es significativa, se enfocará el análisis en estadístico $t$ asociado a cada parámetro en el modelo (cada variable dummy tiene un parámetro asociado).

\section{Variable dependiente:}

$P S=$ Puntaje obtenido en las pruebas Saber Pro para cada uno de los módulos genéricos evaluados (Módulo de Comunicación Escrita, Módulo de Inglés, Módulo de Lectura Crítica, Módulo de Razonamiento Cuantitativo, Módulo de Competencias Ciudadanas).

\section{Variables independientes:}

Comunicaciones en Estadística, diciembre 2019, Vol. 12, No. 2 
116Manuel Arturo Jiménez Ramirez, Edward Parra \& Marianela Luzardo Briceño

Tabla 2: Definición de variables independientes

\begin{tabular}{|c|c|c|}
\hline \multicolumn{2}{|c|}{ Variable } & \multirow[t]{2}{*}{ Definición } \\
\hline $\mathrm{C}$ & Clúster & \\
\hline $\mathbf{I}$ & Institución & $1710,1723,1727,1730$ \\
\hline $\mathbf{S}$ & Semestre & $\begin{array}{l}\text { 20121, 20123, 20131, 20133, } \\
20142,20143,20153\end{array}$ \\
\hline $\mathbf{E}$ & Estrato & $\begin{array}{l}1,2,3,4,5,6,8 \text { (vive en una zona rural } \\
\text { donde no hay estratificación económica) }\end{array}$ \\
\hline GR & Grupo de referencia & $\begin{array}{l}\text { Administración de afines } \\
\text { Arquitectura y urbanismo } \\
\text { Bellas artes y diseño } \\
\text { Ciencias sociales } \\
\text { Comunicación, periodismo y publicidad } \\
\text { Derecho } \\
\text { Economia } \\
\text { Educación } \\
\text { Enfermería } \\
\text { Humanidades } \\
\text { Ingeniería } \\
\text { Medicina } \\
\text { Psicología } \\
\text { Tecnologico en ingeniería, industria y minas }\end{array}$ \\
\hline $\begin{array}{c}\text { EM/EP } \\
\text { Educación de la } \\
\text { madre/ } \\
\text { Educación del } \\
\text { padre }\end{array}$ & $\begin{array}{c}0 \\
1 \\
2 \\
3 \\
4 \\
5 \\
6 \\
7 \\
8 \\
9 \\
10 \\
11 \\
12 \\
13 \\
14 \\
15 \\
16 \\
17 \\
99\end{array}$ & $\begin{array}{l}\text { Ninguno } \\
\text { No tuvo escuela } \\
\text { Preescolar } \\
\text { Básica primaria } \\
\text { Básica secundaria } \\
\text { Media vocacional } \\
\text { Tecnológico o técnico } \\
\text { Universitario } \\
\text { Postgrado } \\
\text { Primaria incompleta } \\
\text { Primaria completa } \\
\text { Secundaria (bachillerato) imcompleta } \\
\text { Secundaria (bachillerato) completa } \\
\text { Educación técnica o tecnológica imcompleta } \\
\text { Educación ténica o tecnológica completa } \\
\text { Educación profesional incompleta } \\
\text { Educación profesional completa } \\
\text { Postgrado } \\
\text { No sabe }\end{array}$ \\
\hline
\end{tabular}

Comunicaciones en Estadística, diciembre 2019, Vol. 12, No. 2 
Los modelos resultantes para analizar las diferencias son los siguientes:

\section{Modelo 1:}

- Entre clústeres, utilizando como categoría de referencia el clúster 1:

$$
P S_{i}=\beta_{1}+\beta_{2} C_{2 i}+\beta_{3} C_{3 i}+e_{i} .
$$

\section{Modelo 2:}

- Entre institución, utilizando como categoría de referencia la institución 1 (institución 1710, central):

$$
P S_{i}=\beta_{1}+\beta_{2} I_{2 i}+\beta_{3} I_{3 i}+\beta_{4} I_{4 i}+e_{i} .
$$

\section{Modelo 3:}

- Entre semestre de presentación de la prueba Saber Pro, utilizando como categoría de referencia el semestre 20123 (segundo semestre del año 2012):

$$
P S_{i}=\beta_{1}+\beta_{2} S_{2 i}+\beta_{3} S_{3 i}+\beta_{4} S_{4 i}+\beta_{5} S_{5 i}+\beta_{6} S_{6 i}+\beta_{7} S_{7 i}+e_{i} .
$$

\section{Modelo 4:}

- Para estrato socioeconómico según factura de energía, utilizando como categoría de referencia el estrato 6:

$$
P S_{i}=\beta_{1}+\beta_{2} E_{2 i}+\beta_{3} E_{3 i}+\beta_{4} E_{4 i}+\beta_{5} E_{5 i}+\beta_{6} E_{6 i}+\beta_{7} E_{7 i}+e_{i} .
$$

\section{Modelo 5:}

- Para grupo de referencia de programas académicos, utilizando como categoría de referencia Ingeniería:

$$
\begin{aligned}
P S_{i}=\beta_{1} & +\beta_{2} G R_{2 i}+\beta_{3} G R_{3 i}+\beta_{4} G R_{4 i}+\beta_{5} G R_{5 i}+\beta_{6} G R_{6 i}+\beta_{7} G_{7 i}+\beta_{8} G_{8 i}+\beta_{9} G_{9 i} \\
& +\beta_{10} G_{10 i}+\beta_{11} 7 G_{117 i}+\beta_{12} G_{12 i}+\beta_{13} G_{13 i}+\beta_{14} G_{14 i}+e_{i} .
\end{aligned}
$$




\section{Resultados}

Se presenta en primer lugar, el resultado del número de clústeres seleccionados, luego el análisis descriptivo de las variables independientes en cada uno de ellos, y, por último, los modelos de regresión que permiten conocer las diferencias significativas entre los puntajes promedio según el caso, a saber, clúster, sede de la institución, semestre de presentación de la prueba, estrato socioeconómico y grupo de referencia.

\subsection{Número de clústeres seleccionados}

Tal como lo muestra la salida del software, con las nueve variables de entrada que se observan en la tabla 1, el usar tres clústeres la calidad de los mismos es correcta.

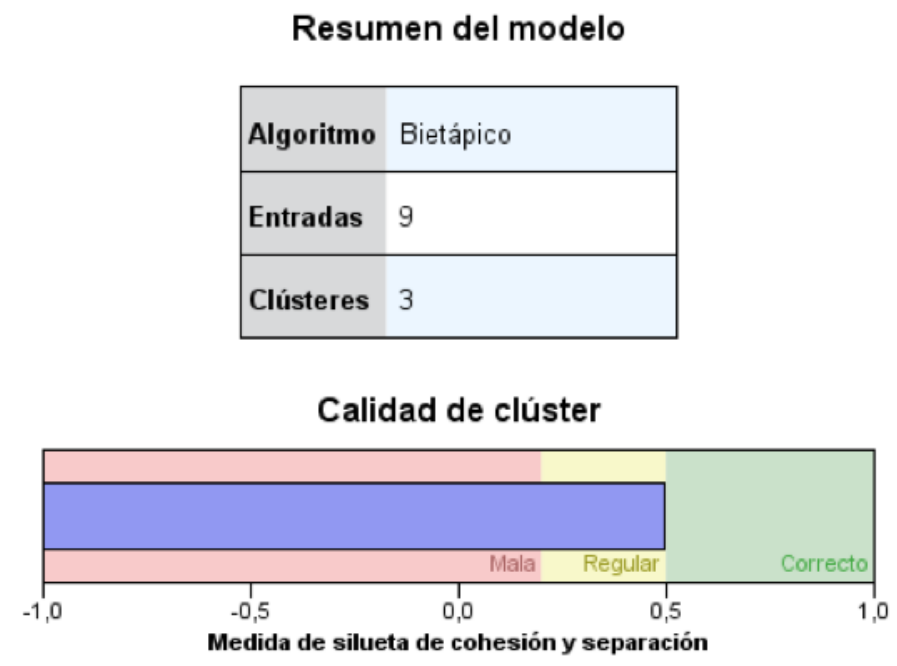

Figura 1: Resultados y selección de número de clústeres.

Fuente: Elaboración propia utilizando SPSS Modeler, 2017 


\subsection{Análisis descriptivo de las variables independientes por clúster}

El análisis descriptivo se realizó con apoyo del software SPSS Modeler, caracterizando variables relacionadas con el estudiante para cada uno de los clústeres seleccionados. Los clústeres obtenidos tienen tamaños diferentes, según la presencia o ausencia de diferentes recursos medidos en la base de datos. El clúster 1 tiene un tamaño de 4.500 registros, el clúster 2 un tamaño de 4.905, y el clúster 3 un tamaño de 2.113.

Tabla 3: Clasificación de estudiantes por clúster según variables socioeconómicas

\begin{tabular}{lccc}
\hline \multicolumn{1}{c}{ Variables } & Clúster $\mathbf{1}$ & Clúster $\mathbf{2}$ & Clúster 3 \\
\hline Econ_sn_internet & 4500 & 4905 & 961 \\
Econ_sn_servicio_tv & 4500 & 4905 & 1010 \\
Econ_sn_telefonia & 4500 & 4380 & 963 \\
Econ_sn_lavadora & 4500 & 4905 & 1076 \\
Econ_sn_horno & 4500 & 3623 & 782 \\
Econ_sn_microondas & 4500 & 3037 & 544 \\
Econ_sn_nevera & 4500 & 4905 & 1794 \\
Econ_sn_automovil & 4500 & 1765 & 446 \\
Econ_sn_reproductordvd & 4500 & 3278 & 896 \\
\hline Número de registros & $\mathbf{4 5 0 0}$ & $\mathbf{4 9 0 5}$ & $\mathbf{2 1 1 3}$ \\
\hline
\end{tabular}

Fuente: Los autores.

Una vez obtenidos los registros se analizó los promedios para cada módulo evaluado en las pruebas Saber Pro, Tabla 4. Se puede apreciar que el clúster 1 tiene promedios más altos, y en todos los módulos, en relación a los clústeres 2 y 3 .

Tabla 4: Promedio por módulos evaluados en las pruebas Saber 11

\begin{tabular}{lccc}
\hline \multicolumn{1}{c}{ Módulo } & Clúster 1 & Clúster 2 & Clúster 3 \\
\hline Comunicación Escrita & 10,31565119 & 10,23246214 & 9,95260116 \\
Inglés & 11,39577199 & 10,88533142 & 10,0247296 \\
Lectura Crítica & 10,58229354 & 10,46536015 & 9,96612903 \\
Razonamiento Cuantitativo & 10,46422801 & 10,32156782 & 9,76110057 \\
Competencias Ciudadanas & 10,30718133 & 10,16449826 & 9,71347249 \\
\hline
\end{tabular}

Fuente: Los autores.

En la Tabla 5 se identifica que la Universidad ha tenido un crecimiento en su población de estudiantes en los últimos dos semestres dentro del intervalo de tiempo analizado. 
120Manuel Arturo Jiménez Ramirez, Edward Parra \& Marianela Luzardo Briceño

Tabla 5: Número de estudiantes por semestre de presentación de la prueba Saber Pro

\begin{tabular}{ccccccc}
\hline Semestre & Clúster $\mathbf{1}$ & $\mathbf{\%}$ & Clúster $\mathbf{2}$ & $\mathbf{\%}$ & Clúster $\mathbf{3}$ & $\mathbf{\%}$ \\
\hline 20121 & 483 & 10,73 & 455 & 9,28 & 143 & 6,77 \\
20123 & 640 & 14,22 & 578 & 11,78 & 189 & 8,94 \\
20131 & 416 & 9,24 & 482 & 9,83 & 240 & 11,36 \\
20133 & 846 & 18,80 & 977 & 19,92 & 361 & 17,08 \\
20142 & 49 & 1,09 & 51 & 1,04 & 14 & 0,66 \\
20143 & 1029 & 22,87 & 1125 & 22,94 & 552 & 26,12 \\
20153 & 1037 & 23,04 & 1237 & 25,22 & 614 & 29,06 \\
\hline
\end{tabular}

Fuente: Los autores.

En relación al estrato socioeconómico se aprecia que la distribución de estrato al que pertenece cada estudiante cambia según el clúster; los estudiantes del clúster 1 se caracterizan por todos tener presencia de los recursos relacionados con las variables de entrada del clúster, y a su vez, la mayor proporción de estudiantes son los pertenecientes a estratos 4 y 5 ; en el clúster 2 se identifica que los estudiantes cuentan con la mayoría de recursos, y son pertenecientes a estrados 3 y 4 ; y en el clúster 3 se ubican los estudiantes que no cuentan con todos los recursos analizados, y además, en su mayoría pertenecen a estratos 1 y 3 .

Tabla 6: Número de estudiantes por estrato socioeconómico

\begin{tabular}{ccccccc}
\hline Estrato & Clúster $\mathbf{1}$ & $\mathbf{\%}$ & Clúster $\mathbf{2}$ & $\mathbf{\%}$ & Clúster $\mathbf{3}$ & $\mathbf{\%}$ \\
\hline 1 & 18 & 0,40 & 116 & 2,36 & 568 & 26,88 \\
2 & 139 & 3,09 & 605 & 12,33 & 476 & 22,53 \\
3 & 730 & 16,22 & 1605 & 32,72 & 538 & 25,46 \\
4 & 1395 & 31,00 & 1596 & 32,54 & 279 & 13,20 \\
5 & 1468 & 32,62 & 802 & 16,35 & 132 & 6,25 \\
6 & 737 & 16,38 & 171 & 3,49 & 28 & 1,33 \\
8 & 9 & 0,20 & 6 & 0,12 & 64 & 3,03 \\
\hline
\end{tabular}

Fuente: Los autores.

En relación al departamento de procedencia la frecuencia muestra que la presencia de estudiantes de diferentes orígenes difiere según el clúster; es decir, y como se observa en la Tabla 7, el departamento de Bolivar y Boyacá no tienen presencial en el clúster 1, Boyacá no tiene presencia en el clúster 2, y el clúster 3 estos dos departamentos sí tienen presencia en este grupo. Hasta este punto se percibe que la distribución de ingresos y los recursos con los que cuenta los estudiantes afecta su desempeño académico, relación que puede apreciarse también, según DANE (2017), por la presencia de departamentos en condiciones de mayor pobreza, en el clúster 3

La educación de los padres es otra variable que se tuvo en cuenta para conocer 
Tabla 7: Número de estudiantes por departamento de origen

\begin{tabular}{lcccccc}
\hline Departamento & Clúster $\mathbf{1}$ & $\mathbf{\%}$ & Clúster $\mathbf{2}$ & $\mathbf{\%}$ & Clúster $\mathbf{3}$ & $\mathbf{\%}$ \\
\hline Amazonas & 0 & 0,00 & 0 & 0,00 & 6 & 0,28 \\
Antioquia & 3117 & 76,87 & 2550 & 51,99 & 699 & 33,08 \\
Arauca & 0 & 0,00 & 0 & 0,00 & 2 & 0,09 \\
Atlántico & 1 & 0,02 & 2 & 0,04 & 0 & 0,00 \\
Bogotá & 33 & 0,81 & 63 & 1,28 & 44 & 2,08 \\
Bolivar & 0 & 0,00 & 1 & 0,02 & 4 & 0,19 \\
Boyacá & 0 & 0,00 & 0 & 0,00 & 3 & 0,14 \\
Caldas & 17 & 0,42 & 30 & 0,61 & 17 & 0,80 \\
Caquetá & 0 & 0,00 & 1 & 0,02 & 24 & 1,14 \\
Casanare & 0 & 0,00 & 0 & 0,00 & 2 & 0,09 \\
Cauca & 2 & 0,05 & 6 & 0,12 & 186 & 8,80 \\
Cesar & 3 & 0,07 & 2 & 0,04 & 2 & 0,09 \\
Chocó & 1 & 0,02 & 0 & 0,00 & 31 & 1,47 \\
Córdoba & 299 & 7,37 & 521 & 10,62 & 283 & 13,39 \\
Cundinamarca & 0 & 0,00 & 4 & 0,08 & 14 & 0,66 \\
Guainía & 0 & 0,00 & 2 & 0,04 & 20 & 0,95 \\
Guaviare & 0 & 0,00 & 0 & 0,00 & 2 & 0,09 \\
Huila & 1 & 0,02 & 1 & 0,02 & 3 & 0,14 \\
La guajira & 4 & 0,10 & 5 & 0,10 & 3 & 0,14 \\
Magdalena & 1 & 0,02 & 1 & 0,02 & 2 & 0,09 \\
Meta & 0 & 0,00 & 2 & 0,04 & 2 & 0,09 \\
Nariño & 0 & 0,00 & 3 & 0,06 & 25 & 1,18 \\
Norte Santander & 2 & 0,05 & 1 & 0,02 & 3 & 0,14 \\
Putumayo & 3 & 0,07 & 5 & 0,10 & 152 & 7,19 \\
Quindío & 1 & 0,02 & 0 & 0,00 & 0 & 0,00 \\
Risaralda & 2 & 0,05 & 4 & 0,08 & 3 & 0,14 \\
San Andrés & 1 & 0,02 & 0 & 0,00 & 0 & 0,00 \\
Santander & 955 & 23,55 & 1568 & 31,97 & 474 & 22,43 \\
Sucre & 4 & 0,10 & 8 & 0,16 & 6 & 0,28 \\
Tolima & 0 & 0,00 & 1 & 0,02 & 4 & 0,19 \\
Valle & 50 & 1,23 & 120 & 2,45 & 80 & 3,79 \\
Vaupés & 0 & 0,00 & 0 & 0,00 & 5 & 0,24 \\
Vichada & 0 & 0,00 & 0 & 0,00 & 10 & 0,47 \\
(en blanco & 3 & 0,07 & 4 & 0,08 & 2 & 0,09 \\
\hline & & & & & &
\end{tabular}

Fuente: Los autores. 
cómo ésta afecta el desempeño del estudiante, en Tabla 8 (educación madre) y Tabla 9 (educación padre). En términos de frecuencia se identificó, y aunque hay porcentajes variados, los estudiantes del clúster 1 tienen padres con educación profesional completa y postgrado; los estudiantes del clúster 2 tienen padres con educación profesional completa y secundaria completa; y los estudiantes del clúster 3 tienen padres con educación primaria incompleta, seguido de estudiantes con padres con educación secundaria completa.

Tabla 8: Número de estudiantes según nivel educativo del padre

\begin{tabular}{ccccccr}
\hline $\begin{array}{l}\text { Educación } \\
\text { Padre }\end{array}$ & Clúster $\mathbf{1}$ & $\mathbf{\%}$ & Clúster $\mathbf{2}$ & $\mathbf{\%}$ & Clúster $\mathbf{3}$ & $\mathbf{\%}$ \\
\hline 0 & & & & & & \\
1 & 34 & 0,76 & 93 & 1,90 & 138 & 6,53 \\
2 & 0 & 0,00 & 0 & 0,00 & 9 & 0,43 \\
3 & 0 & 0,00 & 0 & 0,00 & 21 & 0,99 \\
4 & 1 & 0,02 & 0 & 0,00 & 6 & 0,28 \\
5 & 0 & 0,00 & 0 & 0,00 & 2 & 0,09 \\
6 & 0 & 0,00 & 0 & 0,00 & 4 & 0,19 \\
7 & 0 & 0,00 & 0 & 0,00 & 0 & 0,00 \\
8 & 0 & 0,00 & 0 & 0,00 & 0 & 0,00 \\
9 & 0 & 0,00 & 0 & 0,00 & 0 & 0,00 \\
10 & 138 & 3,07 & 331 & 6,75 & 465 & 22,01 \\
11 & 119 & 2,64 & 319 & 6,50 & 247 & 11,69 \\
12 & 297 & 6,60 & 474 & 9,66 & 188 & 8,90 \\
13 & 748 & 16,62 & 1059 & 21,59 & 395 & 18,69 \\
14 & 114 & 2,53 & 134 & 2,73 & 51 & 2,41 \\
15 & 188 & 10,84 & 602 & 12,27 & 130 & 6,15 \\
16 & 197 & 4,38 & 212 & 4,32 & 50 & 2,37 \\
17 & 8787 & 33,04 & 1159 & 23,63 & 295 & 13,96 \\
99 & 0 & 19,49 & 522 & 10,64 & 112 & 5,30 \\
\hline
\end{tabular}

Fuente: Los autores.

Se aprecia en la Tabla 10 que en los tres cluster la presencia femenina es superior a la masculina en al menos 7.2

En relación a las instituciones, se puede apreciar que la que tiene mayor número de estudiantes es la 1710, Tabla 11, ya que del total de la base de datos el $62.98 \%$ de los registros le pertenecen; por esta razón es la que mayor presencia tiene en cada clúster seleccionado; en función a esto se analizó el porcentaje que corresponde según el número de estudiantes por institución y no para cada clúster, lo cual resultó en que de mayor presencia en el clúster 1 son las instituciones 1710 y 1023, en el clúster 2 se encuentran las 1723 y 1730, y en el clúster 3 las 1727 y 1730.

Con respecto al grupo de referencia se identifica que los programas de Ingeniería tienen un mayor porcentaje de estudiantes en los clústeres 1 y 2 , y los programas 
Tabla 9: Número de estudiantes según nivel educativo de la madre

\begin{tabular}{ccccccc}
\hline $\begin{array}{l}\text { Educación } \\
\text { Madre }\end{array}$ & Clúster $\mathbf{1}$ & $\mathbf{\%}$ & Clúster 2 & $\mathbf{\%}$ & Clúster $\mathbf{3}$ & $\mathbf{\%}$ \\
\hline 0 & 11 & 0,24 & 37 & 0,75 & 111 & 5,25 \\
1 & 0 & 0,00 & 0 & 0,00 & 12 & 0,57 \\
2 & 0 & 0,00 & 0 & 0,00 & 21 & 0,99 \\
3 & 1 & 0,02 & 0 & 0,00 & 6 & 0,28 \\
4 & 0 & 0,00 & 0 & 0,00 & 1 & 0,05 \\
5 & 0 & 0,00 & 0 & 0,00 & 1 & 0,05 \\
6 & 0 & 0,00 & 0 & 0,00 & 1 & 0,05 \\
7 & 0 & 0,00 & 0 & 0,00 & 0 & 0,00 \\
8 & 0 & 0,00 & 0 & 0,00 & 0 & 0,00 \\
9 & 65 & 1,44 & 212 & 4,32 & 389 & 18,41 \\
10 & 84 & 1,87 & 223 & 4,55 & 248 & 11,74 \\
11 & 282 & 6,27 & 435 & 8,87 & 193 & 9,13 \\
12 & 923 & 20,51 & 1229 & 25,06 & 425 & 20,11 \\
13 & 138 & 3,07 & 175 & 3,57 & 50 & 2,37 \\
14 & 675 & 15,00 & 707 & 14,41 & 185 & 8,76 \\
15 & 214 & 4,76 & 185 & 3,77 & 37 & 1,75 \\
16 & 1375 & 30,56 & 1132 & 23,08 & 300 & 14,20 \\
17 & 719 & 15,98 & 553 & 11,27 & 123 & 5,82 \\
99 & 13 & 0,29 & 17 & 0,35 & 10 & 0,47 \\
\hline
\end{tabular}

Fuente: Los autores.

Tabla 10: Número de estudiantes por género

\begin{tabular}{ccccccc}
\hline Género & Clúster $\mathbf{1}$ & $\mathbf{\%}$ & Clúster $\mathbf{2}$ & $\mathbf{\%}$ & Clúster $\mathbf{3}$ & $\mathbf{\%}$ \\
\hline $\mathrm{M}$ & 1958 & 43,51 & 2101 & 42,83 & 978 & 46,28 \\
$\mathrm{~F}$ & 2539 & 56,42 & 2800 & 57,08 & 1130 & 53,48 \\
(En blanco) & 3 & 0,07 & 4 & 0,08 & 5 & 0,24 \\
\hline
\end{tabular}

Fuente: Los autores. 
124Manuel Arturo Jiménez Ramirez, Edward Parra \& Marianela Luzardo Briceño

Tabla 11: Número de estudiantes en relación al total clúster e institución

\begin{tabular}{ccccc}
\hline Clúster & Institución & $\begin{array}{l}\text { Número } \\
\text { de estu- } \\
\text { diantes }\end{array}$ & $\begin{array}{l}\text { Relación } \\
\text { por total } \\
\text { clúster }\end{array}$ & $\begin{array}{l}\text { Relación } \\
\text { por total } \\
\text { Institu- } \\
\text { ción }\end{array}$ \\
\hline \multirow{2}{*}{1} & 1710 & 3207 & $71,27 \%$ & $44,07 \%$ \\
& 1723 & 967 & $21,49 \%$ & $31,77 \%$ \\
& 1727 & 300 & $6,67 \%$ & $26,69 \%$ \\
& 1730 & 26 & $0,58 \%$ & $23,85 \%$ \\
\hline \multirow{2}{*}{2} & 1710 & 2723 & $55,51 \%$ & $37,42 \%$ \\
& 1723 & 1587 & $32,35 \%$ & $52,14 \%$ \\
& 1727 & 532 & $10,85 \%$ & $47,33 \%$ \\
3 & 1730 & 63 & $1,28 \%$ & $57,80 \%$ \\
\hline \multirow{2}{*}{ (en blanco) } & 1710 & 1321 & $62,52 \%$ & $18,15 \%$ \\
& 1723 & 480 & $22,72 \%$ & $15,77 \%$ \\
& 1727 & 292 & $13,82 \%$ & $25,98 \%$ \\
& 1730 & 20 & $0,95 \%$ & $18,35 \%$ \\
\hline
\end{tabular}

Fuente: Los autores.

relacionados con Educación tienen mayor presencia en el clúster 3.

Tabla 12: Número de estudiantes por grupo de referencia

\begin{tabular}{|c|c|c|c|c|c|c|}
\hline Grupo de referencia & Clúster 1 & $\%$ & Clúster 2 & $\%$ & Clúster 3 & $\%$ \\
\hline ADMINISTRACIÓN Y AFINES & 605 & 13,44 & 591 & 12,05 & 161 & 7,62 \\
\hline ARQUITECTURA Y URBANISMO & 373 & 8,29 & 200 & 4,08 & 55 & 2,60 \\
\hline BELLAS ARTES Y DISË̈ $; \frac{1}{2} \mathrm{O}$ & 615 & 13,67 & 385 & 7,85 & 76 & 3,60 \\
\hline CIENCIAS SOCIALES & 74 & 1,64 & 82 & 1,67 & 23 & 1,09 \\
\hline COMUNICACIÓN, PERIODISMO Y PUBLICIDAD & 580 & 12,89 & 513 & 10,46 & 134 & 6,34 \\
\hline DERECHO & 417 & 9,27 & 345 & 7,03 & 121 & 5,73 \\
\hline ECONOMIA & 48 & 1,07 & 83 & 1,69 & 30 & 1,42 \\
\hline EDUCACIÓN & 73 & 1,62 & 307 & 6,26 & 744 & 35,21 \\
\hline ENFERMERÍA & 106 & 2,36 & 207 & 4,22 & 27 & 1,28 \\
\hline HUMANIDADES & 56 & 1,24 & 99 & 2,02 & 105 & 4,97 \\
\hline INGENIERÍA & 1126 & 25,02 & 1606 & 32,74 & 514 & 24,33 \\
\hline MEDICINA & 210 & 4,67 & 141 & 2,87 & 21 & 0,99 \\
\hline PSICOLOGÍA & 216 & 4,80 & 345 & 7,03 & 101 & 4,78 \\
\hline $\begin{array}{l}\text { TECNOLOGICO EN INGENIERÍA, INDUSTRIA Y MI- } \\
\text { NAS }\end{array}$ & 1 & 0,02 & 1 & 0,02 & 1 & 0,05 \\
\hline
\end{tabular}

$\mathrm{Al}$ aplicar los modelos de regresión descritos en la metodología, para conocer si existe o no diferencias significativas para cada módulo entre clústeres, entre institución, entre semestre de presentación, entre, estrato y, entre grupo de referencia, se obtuvo: 


\section{Modelo 1: para clúster}

Tabla 13: Parámetros de regresión por módulo prueba Saber Pro

\begin{tabular}{|c|c|c|c|c|c|c|}
\hline \multicolumn{2}{|c|}{ Variable dependiehtoxdelo } & \multicolumn{2}{|c|}{$\begin{array}{l}\text { Coeficientes no es- } \\
\text { tandarizados }\end{array}$} & \multirow{2}{*}{$\begin{array}{l}\text { Coeficientes } \\
\text { estanda- } \\
\text { rizados } \\
\text { Beta }\end{array}$} & \multirow{2}{*}{$\mathrm{t}$} & \multirow[t]{2}{*}{ Sig. } \\
\hline & & B & $\begin{array}{l}\text { Error } \\
\text { estándar }\end{array}$ & & & \\
\hline Módulo & (Constante) & 10,316 & 0,015 & - & 667,261 & 0 \\
\hline Comunicación & 2 & $-0,083$ & 0,021 & $-0,04$ & $-3,888$ & 0 \\
\hline Escrita & 3 & $-0,363$ & 0,027 & $-0,136$ & $-13,281$ & 0 \\
\hline Módulo & (Constante) & 11,396 & 0,027 & $-\ddot{i} i \frac{1}{2}$ & 426,95 & 0 \\
\hline \multirow[t]{2}{*}{ Inglés } & 2 & $-0,51$ & 0,037 & $-0,137$ & $-13,822$ & 0 \\
\hline & 3 & $-1,371$ & 0,047 & $-0,288$ & $-29,11$ & 0 \\
\hline Módulo & (Constante) & 10,582 & 0,018 & - & 580,53 & 0 \\
\hline \multirow[t]{3}{*}{ Lectura crítica } & 2 & $-0,117$ & 0,025 & $-0,047$ & $-4,636$ & 0 \\
\hline & 3 & $-0,616$ & 0,032 & $-0,193$ & $-19,155$ & 0 \\
\hline & (Constante) & 10,464 & 0,019 & $\dddot{i} i i^{-}-$ & 545,035 & 0 \\
\hline Módulo & 2 & $-0,143$ & 0,027 & $-0,054$ & $-5,37$ & 0 \\
\hline Razonamiento & 3 & $-0,703$ & 0,034 & $-0,209$ & $-20,754$ & 0 \\
\hline \multicolumn{7}{|l|}{ Cuantitativo } \\
\hline & (Constante) & 10,307 & 0,018 & $-\ddot{i} \dot{i} \frac{1}{2}$ & 560,019 & 0 \\
\hline Módulo & 2 & $-0,143$ & 0,025 & $-0,057$ & $-5,603$ & 0 \\
\hline Competencias & 3 & $-0,594$ & 0,032 & $-0,185$ & $-18,28$ & 0 \\
\hline Ciudadanas & & & & & & \\
\hline
\end{tabular}

\section{Modelo 2: para institución}


126Manuel Arturo Jiménez Ramirez, Edward Parra \& Marianela Luzardo Briceño

Tabla 14: Parámetros de regresión por módulo prueba Saber Pro

\begin{tabular}{|c|c|c|c|c|c|c|}
\hline \multirow[t]{2}{*}{ Variable dependiente: } & \multirow[t]{2}{*}{ Modelo } & \multicolumn{2}{|c|}{$\begin{array}{l}\text { Coeficientes no es- } \\
\text { tandarizados }\end{array}$} & \multirow{2}{*}{$\begin{array}{l}\text { Coeficientes } \\
\text { estandariza- } \\
\text { dos } \\
\text { Beta }\end{array}$} & \multirow[t]{2}{*}{$\mathrm{t}$} & \multirow[t]{2}{*}{ Sig. } \\
\hline & & B & $\begin{array}{l}\text { Error } \\
\text { estándar }\end{array}$ & & & \\
\hline \multirow{4}{*}{ MóduloComunicaciónEscrita } & (Constante) & 10,268 & 0,012 & - & 838,427 & 0,000 \\
\hline & 1723 & $-0,075$ & 0,022 & $-0,032$ & $-3,369$ & 0,001 \\
\hline & 1727 & $-0,340$ & 0,033 & $-0,097$ & $-10,196$ & 0,000 \\
\hline & 1730 & $-0,134$ & 0,100 & $-0,013$ & $-1,343$ & 0,179 \\
\hline \multirow{4}{*}{ MóduloInglés } & (Constante) & 11,051 & 0,022 & - & 511,910 & 0,000 \\
\hline & 1723 & $-0,120$ & 0,040 & $-0,029$ & $-3,026$ & 0,002 \\
\hline & 1727 & $-0,799$ & 0,059 & $-0,129$ & $-13,612$ & 0,000 \\
\hline & 1730 & $-1,011$ & 0,177 & $-0,053$ & $-5,721$ & 0,000 \\
\hline \multirow{4}{*}{ MóduloLecturaCrítica } & (Constante) & 10,451 & 0,014 & - & 720,953 & 0,000 \\
\hline & 1723 & 0,045 & 0,027 & 0,016 & 1,687 & 0,092 \\
\hline & 1727 & $-0,406$ & 0,039 & $-0,098$ & $-10,296$ & 0,000 \\
\hline & 1730 & $-0,158$ & 0,119 & $-0,012$ & $-1,330$ & 0,183 \\
\hline \multirow{4}{*}{ MóduloRazonamiento Cuantitativo } & (Constante) & 10,197 & 0,015 & - & 671,760 & 0,000 \\
\hline & 1723 & 0,402 & 0,028 & 0,136 & 14,443 & 0,000 \\
\hline & 1727 & $-0,238$ & 0,041 & $-0,054$ & $-5,770$ & 0,000 \\
\hline & 1730 & $-0,553$ & 0,124 & $-0,041$ & $-4,449$ & 0,000 \\
\hline \multirow{4}{*}{ Mï $¿ \frac{1}{2}$ duloCompetencias Ciudadanas } & (Constante) & 10,165 & 0,015 & - & 693,193 & 0,000 \\
\hline & 1723 & 0,018 & 0,027 & 0,007 & 0,686 & 0,493 \\
\hline & 1727 & $-0,276$ & 0,040 & $-0,066$ & $-6,923$ & 0,000 \\
\hline & 1730 & $-0,290$ & 0,120 & $-0,023$ & $-2,418$ & 0,016 \\
\hline
\end{tabular}

En relación a la institución, se puede observar que la que tuvo mejor desempeño es la 1710 en la mayoría de los módulos, con excepción de razonamiento cuantitativo y competencias ciudadanas en la que la 1723 sobresale con resultados superiores a los de la comparación.

\section{Modelo 3: para semestre de presentación de la prueba Saber Pro}

Analizando el semestre de presentación se aprecia que el semestre en el que los estudiantes presentaron mejores desempeños en los módulos de la prueba Saber Pro fue en el periodo 20123, aunque, como se observa en la Tabla 15, unos semestres parecen mostrar que no hay diferencia significativa en relación al semestre de comparación. 
Tabla 15: Parámetros de regresión por módulo prueba Saber Pro

\begin{tabular}{|c|c|c|c|c|c|c|}
\hline \multirow[t]{2}{*}{ Variable dependiente: } & \multirow[t]{2}{*}{ Modelo } & \multicolumn{2}{|c|}{$\begin{array}{l}\text { Coeficientes no es- } \\
\text { tandarizados }\end{array}$} & \multirow{2}{*}{$\begin{array}{l}\text { Coeficientes } \\
\text { estandariza- } \\
\text { dos } \\
\text { Beta }\end{array}$} & \multirow[t]{2}{*}{$\mathrm{t}$} & \multirow[t]{2}{*}{ Sig. } \\
\hline & & B & $\begin{array}{l}\text { Error } \\
\text { estándar }\end{array}$ & & & \\
\hline \multirow{7}{*}{ Módulo Comunicación Escrita } & (constante) & 10,531 & 0,028 & - & 370,203 & 0,000 \\
\hline & 20121 & $-0,029$ & 0,042 & $-0,008$ & $-0,682$ & 0,495 \\
\hline & 20131 & $-0,530$ & 0,042 & $-0,154$ & $-12,725$ & 0,000 \\
\hline & 20133 & $-0,334$ & 0,036 & $-0,127$ & $-9,304$ & 0,000 \\
\hline & 20142 & $-0,612$ & 0,101 & $-0,058$ & $-6,053$ & 0,000 \\
\hline & 20143 & $-0,407$ & 0,035 & $-0,168$ & $-11,768$ & 0,000 \\
\hline & 20153 & $-0,375$ & 0,034 & $-0,157$ & $-10,942$ & 0,000 \\
\hline \multirow{7}{*}{ Módulo Inglés } & (Constante) & 11,152 & 0,051 & - & 220,330 & 0,000 \\
\hline & 20121 & $-0,178$ & 0,076 & $-0,028$ & $-2,357$ & 0,018 \\
\hline & 20131 & $-0,484$ & 0,074 & $-0,078$ & $-6,496$ & 0,000 \\
\hline & 20133 & $-0,197$ & 0,064 & $-0,042$ & $-3,065$ & 0,002 \\
\hline & 20142 & 0,448 & 0,161 & 0,027 & 2,785 & 0,005 \\
\hline & 20143 & $-0,240$ & 0,062 & $-0,055$ & $-3,890$ & 0,000 \\
\hline & 20153 & $-0,269$ & 0,061 & $-0,063$ & $-4,393$ & 0,000 \\
\hline \multirow{7}{*}{ Módulo Lectura Crítica } & (Constante) & 10,435 & 0,034 & - & 308,671 & 0,000 \\
\hline & 20121 & 0,136 & 0,050 & 0,032 & 2,689 & 0,007 \\
\hline & 20131 & $-0,197$ & 0,050 & $-0,048$ & $-3,960$ & 0,000 \\
\hline & 20133 & $-0,049$ & 0,043 & $-0,016$ & $-1,152$ & 0,249 \\
\hline & 20142 & 0,110 & 0,107 & 0,010 & 1,024 & 0,306 \\
\hline & 20143 & $-0,122$ & 0,041 & $-0,042$ & $-2,962$ & 0,003 \\
\hline & 20153 & 0,120 & 0,041 & 0,042 & 2,927 & 0,003 \\
\hline \multirow{7}{*}{ Módulo Razonamiento Cuantitativo } & (Constante) & 10,374 & 0,036 & - & 290,038 & 0,000 \\
\hline & 20121 & $-0,043$ & 0,053 & $-0,010$ & $-0,801$ & 0,423 \\
\hline & 20131 & $-0,300$ & 0,053 & $-0,069$ & $-5,691$ & 0,000 \\
\hline & 20133 & $-0,081$ & 0,045 & $-0,024$ & $-1,780$ & 0,075 \\
\hline & 20142 & 0,291 & 0,114 & 0,025 & 2,566 & 0,010 \\
\hline & 20143 & $-0,120$ & 0,044 & $-0,039$ & $-2,751$ & 0,006 \\
\hline & 20153 & $-0,099$ & 0,043 & $-0,033$ & $-2,284$ & 0,022 \\
\hline \multirow{7}{*}{ Módulo Competencias Ciudadanas } & (Constante) & 10,348 & 0,034 & - & 304,317 & 0,000 \\
\hline & 20121 & 0,098 & 0,051 & 0,023 & 1,932 & 0,053 \\
\hline & 20131 & $-0,379$ & 0,050 & $-0,091$ & $-7,565$ & 0,000 \\
\hline & 20133 & $-0,266$ & 0,043 & $-0,084$ & $-6,162$ & 0,000 \\
\hline & 20142 & $-0,006$ & 0,108 & $-0,001$ & $-0,056$ & 0,955 \\
\hline & 20143 & $-0,340$ & 0,042 & $-0,116$ & $-8,201$ & 0,000 \\
\hline & 20153 & $-0,197$ & 0,041 & $-0,068$ & $-4,789$ & 0,000 \\
\hline
\end{tabular}

\section{Modelo 4: para estrato socioeconómico según factura de energía eléctrica}

Con respecto al estrato socioeconómico, Tabla 16, se aprecia que los estudiantes con mejor desempeño son los estudiantes con nivel 6 de estrato socioeconómico, aunque en algunos casos se observa que para el nivel 5 la diferencia no es significativa. 
128Manuel Arturo Jiménez Ramirez, Edward Parra \& Marianela Luzardo Briceño

Tabla 16: Parámetros de regresión por módulo prueba Saber Pro

\begin{tabular}{|c|c|c|c|c|c|c|}
\hline \multirow[t]{2}{*}{ Variable dependiente: } & \multirow[t]{2}{*}{ Modelo } & \multicolumn{2}{|c|}{$\begin{array}{l}\text { Coeficientes no es- } \\
\text { tandarizados }\end{array}$} & \multirow{2}{*}{$\begin{array}{l}\text { Coeficientes } \\
\text { estandariza- } \\
\text { dos } \\
\text { Beta }\end{array}$} & \multirow[t]{2}{*}{$\mathrm{t}$} & \multirow[t]{2}{*}{ Sig. } \\
\hline & & B & $\begin{array}{l}\text { Error } \\
\text { estándar }\end{array}$ & & & \\
\hline \multirow{7}{*}{ Módulo Comunicación Escrita } & (Constante) & 10,327 & 0,033 & - & 308,476 & 0,000 \\
\hline & 1 & $-0,746$ & 0,051 & $-0,173$ & $-14,557$ & 0,000 \\
\hline & 2 & $-0,193$ & 0,045 & $-0,058$ & $-4,339$ & 0,000 \\
\hline & 3 & $-0,053$ & 0,039 & $-0,022$ & $-1,366$ & 0,172 \\
\hline & 4 & $-0,071$ & 0,038 & $-0,031$ & $-1,862$ & 0,063 \\
\hline & 5 & $-0,023$ & 0,040 & $-0,009$ & $-0,570$ & 0,568 \\
\hline & 6 & $-0,878$ & 0,122 & $-0,069$ & $-7,187$ & 0,000 \\
\hline \multirow{7}{*}{ Módulo Inglés } & (Constante) & 12,054 & 0,057 & - & 212,093 & 0,000 \\
\hline & 1 & $-2,668$ & 0,087 & $-0,348$ & $-30,808$ & 0,000 \\
\hline & 2 & $-1,848$ & 0,075 & $-0,309$ & $-24,489$ & 0,000 \\
\hline & 3 & $-1,415$ & 0,065 & $-0,333$ & $-21,644$ & 0,000 \\
\hline & 4 & $-0,938$ & 0,064 & $-0,230$ & $-14,561$ & 0,000 \\
\hline & 5 & $-0,593$ & 0,067 & $-0,130$ & $-8,850$ & 0,000 \\
\hline & 6 & $-2,498$ & 0,204 & $-0,112$ & $-12,239$ & 0,000 \\
\hline \multirow{7}{*}{ Módulo Lectura Crítica } & (Constante) & 10,691 & 0,039 & - & 271,695 & 0,000 \\
\hline & 1 & $-1,285$ & 0,060 & $-0,250$ & $-21,438$ & 0,000 \\
\hline & 2 & $-0,477$ & 0,052 & $-0,119$ & $-9,130$ & 0,000 \\
\hline & 3 & $-0,274$ & 0,045 & $-0,096$ & $-6,062$ & 0,000 \\
\hline & 4 & $-0,143$ & 0,045 & $-0,052$ & $-3,216$ & 0,001 \\
\hline & 5 & $-0,101$ & 0,046 & $-0,033$ & $-2,181$ & 0,029 \\
\hline & 6 & $-1,170$ & 0,141 & $-0,078$ & $-8,282$ & 0,000 \\
\hline \multirow{7}{*}{ Módulo Razonamiento Cuantitativo } & (Constante) & 10,536 & 0,042 & - & 253,231 & 0,000 \\
\hline & 1 & $-1,278$ & 0,063 & $-0,235$ & $-20,151$ & 0,000 \\
\hline & 2 & $-0,534$ & 0,055 & $-0,126$ & $-9,660$ & 0,000 \\
\hline & 3 & $-0,303$ & 0,048 & $-0,101$ & $-6,333$ & 0,000 \\
\hline & 4 & $-0,082$ & 0,047 & $-0,028$ & $-1,739$ & 0,082 \\
\hline & 5 & $-0,078$ & 0,049 & $-0,024$ & $-1,597$ & 0,110 \\
\hline & 6 & $-1,198$ & 0,149 & $-0,076$ & $-8,014$ & 0,000 \\
\hline \multirow{7}{*}{ Módulo Competencias Ciudadanas } & (Constante) & 10,390 & 0,040 & - & 260,393 & 0,000 \\
\hline & 1 & $-1,171$ & 0,061 & $-0,226$ & $-19,268$ & 0,000 \\
\hline & 2 & $-0,437$ & 0,053 & $-0,108$ & $-8,245$ & 0,000 \\
\hline & 3 & $-0,244$ & 0,046 & $-0,085$ & $-5,307$ & 0,000 \\
\hline & 4 & $-0,165$ & 0,045 & $-0,060$ & $-3,640$ & 0,000 \\
\hline & 5 & $-0,075$ & 0,047 & $-0,025$ & $-1,598$ & 0,110 \\
\hline & 6 & $-1,011$ & 0,143 & $-0,067$ & $-7,058$ & 0,000 \\
\hline
\end{tabular}

\section{Modelo 5: para grupo de referencia de programas académicos}

Por último, en cuanto a grupo de referencia las significancias cambian según el módulo analizado. El programa de ingeniería muestra ser más fuerte en los módulos de razonamiento cuantitativo y competencias ciudadanas, y medicina presenta mejores resultados en los módulos de lectura crítica, inglés y comunicación escrita. 
Tabla 17: Parámetros de regresión por módulo prueba Saber Pro

\begin{tabular}{|c|c|c|c|c|c|c|}
\hline \multirow[t]{2}{*}{ Variable dependiente: } & \multirow[t]{2}{*}{ Modelo } & \multicolumn{2}{|c|}{$\begin{array}{l}\text { Coeficientes no es- } \\
\text { tandarizados }\end{array}$} & \multirow{2}{*}{$\begin{array}{l}\text { Coeficientes } \\
\text { estandariza- } \\
\text { dos } \\
\text { Beta }\end{array}$} & \multirow[t]{2}{*}{$\mathbf{t}$} & \multirow[t]{2}{*}{ Sig. } \\
\hline & & B & $\begin{array}{l}\text { Error } \\
\text { estándar }\end{array}$ & & & \\
\hline \multirow{14}{*}{ Módulo Comunicación Escrita } & (Constante) & 10,107 & 0,018 & - & 567,623 & 0,000 \\
\hline & Administración & 0,058 & 0,033 & 0,018 & 1,752 & 0,080 \\
\hline & Arquitectura & 0,098 & 0,044 & 0,022 & 2,231 & 0,026 \\
\hline & Bellas artes & 0,088 & 0,036 & 0,025 & 2,477 & 0,013 \\
\hline & Ciencias sociales & 0,508 & 0,078 & 0,061 & 6,515 & 0,000 \\
\hline & Comunicación & 0,310 & 0,034 & 0,092 & 9,067 & 0,000 \\
\hline & Derecho & 0,375 & 0,039 & 0,096 & 9,690 & 0,000 \\
\hline & Economía & 0,089 & 0,082 & 0,010 & 1,084 & 0,279 \\
\hline & Educación & $-0,398$ & 0,035 & $-0,114$ & $-11,308$ & 0,000 \\
\hline & Enfermería & 0,245 & 0,057 & 0,040 & 4,256 & 0,000 \\
\hline & Humanidades & 0,712 & 0,065 & 0,103 & 10,914 & 0,000 \\
\hline & Medicina & 0,574 & 0,055 & 0,099 & 10,384 & 0,000 \\
\hline & Psicología & 0,220 & 0,043 & 0,049 & 5,059 & 0,000 \\
\hline & Tecnología & 1,127 & 0,582 & 0,018 & 1,936 & 0,053 \\
\hline \multirow{14}{*}{ Módulo Inglés } & (Constante) & 11,103 & 0,031 & - & 356,511 & 0,000 \\
\hline & Administración & $-0,045$ & 0,057 & $-0,008$ & $-0,775$ & 0,438 \\
\hline & Arquitectura & 0,252 & 0,077 & 0,031 & 3,285 & 0,001 \\
\hline & Bellas artes & 0,330 & 0,062 & 0,052 & 5,303 & 0,000 \\
\hline & Ciencias sociales & $-0,400$ & 0,137 & $-0,027$ & $-2,927$ & 0,003 \\
\hline & Comunicación & $-0,051$ & 0,060 & $-0,008$ & $-0,849$ & 0,396 \\
\hline & Derecho & $-0,213$ & 0,067 & $-0,031$ & $-3,161$ & 0,002 \\
\hline & Economía & $-0,393$ & 0,143 & $-0,025$ & $-2,758$ & 0,006 \\
\hline & Educación & $-1,397$ & 0,061 & $-0,225$ & $-22,768$ & 0,000 \\
\hline & Enfermería & $-0,943$ & 0,101 & $-0,087$ & $-9,350$ & 0,000 \\
\hline & Humanidades & $-0,909$ & 0,114 & $-0,073$ & $-7,965$ & 0,000 \\
\hline & Medicina & 1,084 & 0,097 & 0,104 & 11,193 & 0,000 \\
\hline & Psicología & $-0,510$ & 0,076 & $-0,064$ & $-6,717$ & 0,000 \\
\hline & Tecnología & 0,330 & 1,023 & 0,003 & 0,322 & 0,747 \\
\hline \multirow{14}{*}{ Módulo Lectura Crítica } & (Constante) & 10,460 & 0,021 & - & 500,535 & 0,000 \\
\hline & Administración & $-0,173$ & 0,039 & $-0,045$ & $-4,483$ & 0,000 \\
\hline & Arquitectura & 0,016 & 0,052 & 0,003 & 0,307 & 0,759 \\
\hline & Bellas artes & 0,003 & 0,042 & 0,001 & 0,062 & 0,951 \\
\hline & Ciencias sociales & 0,154 & 0,092 & 0,015 & 1,683 & 0,092 \\
\hline & Comunicación & 0,200 & 0,040 & 0,050 & 4,993 & 0,000 \\
\hline & Derecho & 0,271 & 0,045 & 0,058 & 5,992 & 0,000 \\
\hline & Economía & $-0,118$ & 0,096 & $-0,011$ & $-1,237$ & 0,216 \\
\hline & Educación & $-0,910$ & 0,041 & $-0,219$ & $-22,109$ & 0,000 \\
\hline & Enfermería & $-0,206$ & 0,068 & $-0,028$ & $-3,050$ & 0,002 \\
\hline & Humanidades & $-0,023$ & 0,077 & $-0,003$ & $-0,300$ & 0,764 \\
\hline & Medicina & 0,819 & 0,065 & 0,117 & 12,597 & 0,000 \\
\hline & Psicología & 0,117 & 0,051 & 0,022 & 2,300 & 0,021 \\
\hline & Tecnología & $-0,260$ & 0,686 & $-0,003$ & $-0,379$ & 0,705 \\
\hline
\end{tabular}

Comunicaciones en Estadística, diciembre 2019, Vol. 12, No. 2 
130Manuel Arturo Jiménez Ramirez, Edward Parra \& Marianela Luzardo Briceño

Tabla 18: Parámetros de regresión por módulo prueba Saber Pro

\begin{tabular}{|c|c|c|c|c|c|c|}
\hline \multirow[t]{2}{*}{ Variable dependiente: } & \multirow[t]{2}{*}{ Modelo } & \multicolumn{2}{|c|}{$\begin{array}{l}\text { Coeficientes no es- } \\
\text { tandarizados }\end{array}$} & \multirow{2}{*}{$\begin{array}{l}\text { Coeficientes } \\
\text { estandariza- } \\
\text { dos } \\
\text { Beta }\end{array}$} & \multirow[t]{2}{*}{$\mathbf{t}$} & \multirow[t]{2}{*}{ Sig. } \\
\hline & & $\mathbf{B}$ & $\begin{array}{l}\text { Error } \\
\text { estándar }\end{array}$ & & & \\
\hline \multirow{14}{*}{ Módulo Razonamiento Cuantitativo } & (Constante) & 10,940 & 0,021 & - & 525,520 & 0,000 \\
\hline & Administración & $-0,607$ & 0,038 & $-0,150$ & $-15,821$ & 0,000 \\
\hline & Arquitectura & $-0,527$ & 0,051 & $-0,092$ & $-10,273$ & 0,000 \\
\hline & Bellas artes & $-0,821$ & 0,042 & $-0,183$ & $-19,711$ & 0,000 \\
\hline & Ciencias sociales & $-1,125$ & 0,091 & $-0,106$ & $-12,308$ & 0,000 \\
\hline & Comunicación & $-1,002$ & 0,040 & $-0,236$ & $-25,105$ & 0,000 \\
\hline & Derecho & $-0,832$ & 0,045 & $-0,169$ & $-18,455$ & 0,000 \\
\hline & Economía & $-0,724$ & 0,095 & $-0,065$ & $-7,596$ & 0,000 \\
\hline & Educación & $-1,777$ & 0,041 & $-0,405$ & $-43,332$ & 0,000 \\
\hline & Enfermería & $-0,940$ & 0,067 & $-0,122$ & $-13,951$ & 0,000 \\
\hline & Humanidades & $-1,145$ & 0,076 & $-0,131$ & $-15,005$ & 0,000 \\
\hline & Medicina & 0,057 & 0,065 & 0,008 & 0,887 & 0,375 \\
\hline & Psicología & $-1,125$ & 0,051 & $-0,200$ & $-22,177$ & 0,000 \\
\hline & Tecnología & $-1,640$ & 0,684 & $-0,020$ & $-2,399$ & 0,016 \\
\hline \multirow{14}{*}{ Módulo Competencias Ciudadanas } & (Constante) & 10,177 & 0,021 & - & 483,941 & 0,000 \\
\hline & Administración & $-0,144$ & 0,039 & $-0,037$ & $-3,715$ & 0,000 \\
\hline & Arquitectura & $-0,037$ & 0,052 & $-0,007$ & $-0,722$ & 0,470 \\
\hline & Bellas artes & $-0,150$ & 0,042 & $-0,035$ & $-3,577$ & 0,000 \\
\hline & Ciencias sociales & 0,396 & 0,092 & 0,039 & 4,290 & 0,000 \\
\hline & Comunicación & 0,130 & 0,040 & 0,032 & 3,215 & 0,001 \\
\hline & Derecho & 0,536 & 0,046 & 0,114 & 11,765 & 0,000 \\
\hline & Economía & $-0,079$ & 0,096 & $-0,008$ & $-0,825$ & 0,410 \\
\hline & Educación & $-0,881$ & 0,041 & $-0,210$ & $-21,274$ & 0,000 \\
\hline & Enfermería & $-0,140$ & 0,068 & $-0,019$ & $-2,060$ & 0,039 \\
\hline & Humanidades & 0,053 & 0,077 & 0,006 & 0,684 & 0,494 \\
\hline & Medicina & 0,792 & 0,065 & 0,113 & 12,108 & 0,000 \\
\hline & Psicología & $-0,004$ & 0,051 & $-0,001$ & $-0,083$ & 0,934 \\
\hline & Tecnología & 0,023 & 0,691 & 0,000 & 0,033 & 0,973 \\
\hline
\end{tabular}

\section{Conclusiones}

Al analizar cada uno de los clústeres se identifica diferencias significativas en los puntajes para cada uno de los módulos, con justificación en el nivel de recursos que posee el estudiante. Se puede concluir que el nivel que posee el estudiante tiene una alta relación con el estrato socioeconómico, y así mismo con la educación de los padres, y en algunos casos con el departamento de procedencia; siendo los estudiantes con mejores recursos lo que presentan mejores resultados en los módulos de la prueba Saber Pro.

La institución 1730 del clúster 3 mostraba una diferencia negativa con respecto a la 1710 del clúster1, pero con una significancia alta lo que indicaba que esta diferencia no parecía ser significativa. Esto puede justificarse debido al tamaño de cada grupo ya que se están comparando 3207 de la institución 1710 del clúster 1, con 20 registros de la sede 1730 del clúster

En análisis para cada uno de los módulos por grupo de referencia otorgan un panorama de las fortalezas de los programas académicos. Destacándose Ingeniería en áreas de razonamiento cuantitativo, justificación que se haya en que estas ca- 
rreras tienen una relación alta con modelos matemáticos, así como resolución de problemas de índole social. Otras de las carreras que sobresalen es la carrera de Medicina con puntajes superiores en áreas relacionadas con pensamiento crítico y comunicación escrita.

Las referencias cruzadas de todas las variables no se tuvieron en cuenta en esta investigación debida que en muchos casos se identificaban casos muy específicos, lo que no permitían hacer comparaciones estadísticas en relación a la base de datos. En este sentido, al trabajar con grupos más grandes, y por tanto, hacer comparaciones facilitó el ejercicio inferencial el cual servirá para realizar planes para mejoramiento continuo y la autoevaluación institucional.

Algunas limitaciones se encontraron en la capacidad de procesamiento de los computadores, aunque se puede entender que el número de relaciones a analizar eran de combinaciones muy grandes lo que excedía la capacidad de las máquinas.

Recomendaciones para futuras investigaciones son las de seguir ampliar la base de datos para cada una de las instituciones. Con esto se espera conocer si las significancias, como es el caso de la sede 1730 del clúster 3, cambian o se mantienen.

Otra de las recomendaciones para futuras investigaciones es la de realizar un análisis por bloques al azar para conocer el porcentaje de explicación en la variación general para cada uno de los puntajes en los módulos de las pruebas Saber Pro

Recibido: Junio de 2019 Aceptado: Febrero de 2020

\section{Referencias}

Alfiani, A. P., Wulandari, F. A. et al. (2015), 'Mapping student's performance based on data mining approach (a case study)', Agriculture and Agricultural Science Procedia 3, 173-177.

Brown, S., White, S. \& Power, N. (2017), 'Introductory anatomy and physiology in an undergraduate nursing curriculum', Advances in physiology education 41(1), 56-61.

Ferguson, T. D., Deephouse, D. L. \& Ferguson, W. L. (2000), 'Do strategic groups differ in reputation?', Strategic Management Journal 21(12), 1195-1214.

Field, S., Kuczera, M. \& Pont, B. (2007), 'No more failures: ten steps to equity in education. summary and policy recommendations'.

for Economic Co-operation, O. \& (OECD), D. (2010), PISA 2009 results: what makes a school successful?: resources, policies and practices (vol. iv), OECD, Paris, France.

Comunicaciones en Estadística, diciembre 2019, Vol. 12, No. 2 
IBM Knowledge Center (2012), 'Log-likelihood distance (twostep clustering algorithms)', Journal of Marketing Research .

*www.ibm.com

OECD, $\ddot{Y}$. (2012), 'Equity and quality in education: Supporting disadvantaged students and schools'.

Punj, G. \& Stewart, D. W. (1983), 'Cluster analysis in marketing research: Review and suggestions for application', Journal of Marketing Research 20(2), 134148.

Reardon, S. F. (2011), 'The widening academic achievement gap between the rich and the poor: New evidence and possible explanations', Whither opportunity pp. 91-116.

Sideridis, G. D., Mouzaki, A., Simos, P. \& Protopapas, A. (2006), 'Classification of students with reading comprehension difficulties: The roles of motivation, affect, and psychopathology', Learning Disability Quarterly 29(3), 159-180.

Zhao, J., Jin, L. \& Shi, L. (2015), 'Mixture model selection via hierarchical bic', Computational Statistics \& Data Analysis 88, 139-153. 